ANNALES

POLONICI MATHEMATICI

LXXIX.2 (2002)

\title{
Linear differential polynomials sharing the same 1-points with weight two
}

\author{
by INDRAJIT LAHIRI (Kalyani)
}

\begin{abstract}
We prove a uniqueness theorem for meromorphic functions involving differential polynomials which improves some previous results and provides a better answer to a question of C. C. Yang.
\end{abstract}

1. Introduction and definitions. Let $f$ and $g$ be two nonconstant meromorphic functions defined in the open complex plane $\mathbb{C}$. If for $a \in \mathbb{C} \cup$ $\{\infty\}, f-a$ and $g-a$ have the same set of zeros with the same multiplicities, we say that $f$ and $g$ share the value a $C M$ (counting multiplicities) and if we do not consider the multiplicities, $f$ and $g$ are said to share the value a $I M$ (ignoring multiplicities). We do not explain the standard notations and definitions of the value distribution theory as those are available in [2].

In [9] C. C. Yang asked: What can be said if two nonconstant entire functions $f, g$ share the value $0 C M$ and their first derivatives share the value 1 CM?

A number of authors have worked on this question of Yang (e.g. [3, 6, $7,10,11]$ ). To answer the question of Yang, K. Shibazaki [7] proved the following result.

TheOREM A. Let $f$ and $g$ be two entire functions of finite order. If $f^{\prime}$ and $g^{\prime}$ share the value $1 C M$ with $\delta(0 ; f)>0$ and 0 being lacunary for $g$ then either $f \equiv g$ or $f^{\prime} g^{\prime} \equiv 1$.

Improving Theorem A, H. X. Yi [12] obtained the following theorem.

THEOREM B. Let $f, g$ be two entire functions such that $f^{(n)}$ and $g^{(n)}$ share the value $1 C M$. If $\delta(0 ; f)+\delta(0 ; g)>1$ then either $f \equiv g$ or $f^{(n)} g^{(n)} \equiv 1$.

For meromorphic functions H. X. Yi and C. C. Yang [13] proved the following result.

2000 Mathematics Subject Classification: Primary 30D35.

Key words and phrases: uniqueness, weighted sharing, differential polynomial. 
THEOREM C. Let $f$ and $g$ be two meromorphic functions such that $\Theta(\infty ; f)=\Theta(\infty ; g)=1$. If $f^{(n)}$ and $g^{(n)}$ share the value $1 C M$ with $\delta(0 ; f)+\delta(0 ; g)>1$ then either $f \equiv g$ or $f^{(n)} g^{(n)} \equiv 1$.

In [3] the following question was asked: What can be said if two linear differential polynomials generated by two meromorphic functions $f$ and $g$ share the value 1 CM?

We denote by $\Psi(D)$ a linear differential operator with constant coefficients of the form

$$
\Psi(D)=\sum_{i=1}^{p} \alpha_{i} D^{i},
$$

where $D=d / d z$.

Also we denote by $N_{k}(r, a ; f)$ the counting function of $a$-points of $f$ where an $a$-point of multiplicity $\mu$ is counted $\mu$ times if $\mu \leq k$ and $k$ times if $\mu>k$, where $k$ is a positive integer. We put

$$
\delta_{k}(a ; f)=1-\limsup _{r \rightarrow \infty} \frac{N_{k}(r, a ; f)}{T(r, f)} .
$$

Clearly $\delta(a ; f) \leq \delta_{k}(a ; f) \leq \delta_{k-1}(a ; f) \leq \ldots \leq \delta_{1}(a ; f)=\Theta(a ; f)$.

In [3] the following two theorems were proved.

TheOREM D. Let $f$ and $g$ be two meromorphic functions such that

(i) $\Psi(D) f, \Psi(D) g$ are nonconstant and share $1 C M$, and

$$
\begin{aligned}
\frac{\sum_{a \neq \infty} \delta(a ; f)}{1+p(1-\Theta(\infty ; f))} & +\frac{\sum_{a \neq \infty} \delta(a ; g)}{1+p(1-\Theta(\infty ; g))} \\
& >1+\frac{4(1-\Theta(\infty ; f))}{\sum_{a \neq \infty} \delta_{p}(a ; f)}+\frac{4(1-\Theta(\infty ; g))}{\sum_{a \neq \infty} \delta_{p}(a ; g)},
\end{aligned}
$$

where $\sum_{a \neq \infty} \delta_{p}(a ; f)>0$ and $\sum_{a \neq \infty} \delta_{p}(a ; g)>0$. Then either $[\Psi(D) f][\Psi(D) g]$ $\equiv 1$ or $f-g \equiv s$ where $s=s(z)$ is a solution of the differential equation $\Psi(D) w=0$.

TheOREM E. If $f$ and $g$ are of finite order then Theorem $\mathrm{D}$ still holds if condition (ii) is replaced by the following weaker one:

$$
\begin{aligned}
\frac{\sum_{a \neq \infty} \delta(a ; f)}{1+p(1-\Theta(\infty ; f))}+\frac{\sum_{a \neq \infty} \delta(a ; g)}{1+p(1-\Theta(\infty ; g))} \\
>1+\frac{2(1-\Theta(\infty ; f))}{\sum_{a \neq \infty} \delta_{p}(a ; f)}+\frac{2(1-\Theta(\infty ; g))}{\sum_{a \neq \infty} \delta_{p}(a ; g)},
\end{aligned}
$$

where $\sum_{a \neq \infty} \delta_{p}(a ; f)>0$ and $\sum_{a \neq \infty} \delta_{p}(a ; g)>0$.

H. X. Yi [10] also answered the question of Yang and proved the following result. 
THEOREM F. Let $f$ and $g$ be two nonconstant entire functions. Assume that $f, g$ share $0 C M$ and $f^{(n)}, g^{(n)}$ share $1 C M$, where $n$ is a nonnegative integer. If $\delta(0 ; f)>1 / 2$ then either $f \equiv g$ or $f^{(n)} g^{(n)} \equiv 1$.

As an application of Theorem D, in [3] the following answer to the question of Yang was given.

THEOREM G. Let $f$ and $g$ be two nonconstant meromorphic functions with $\Theta(\infty ; f)=\Theta(\infty ; g)=1$. Suppose that $f^{(n)}, g^{(n)}(n \geq 1)$ share $1 C M$ and $f, g$ share a value $b(\neq \infty)$ IM. If $\sum_{a \neq \infty} \delta(a ; f)+\sum_{a \neq \infty} \delta(a ; g)>1$ then either $f \equiv g$ or $f^{(n)} g^{(n)} \equiv 1$.

The following example shows that in Theorems D and E sharing the value 1 cannot be relaxed from CM to IM.

ExAmple 1. Let $f=-i e^{z}, g=2^{-p} e^{2 z}-2 i e^{z}$ and $\Psi(D)=D^{p}$. Then $\Psi(D) f, \Psi(D) g$ share the value $1 \mathrm{IM}$ and $\sum_{a \neq \infty} \delta(a ; f)+\sum_{a \neq \infty} \delta(a ; g)=3 / 2$ but neither $f \equiv g+Q$ nor $[\Psi(D) f][\Psi(D) g] \equiv 1$ where $Q$ is a polynomial of degree at most $p-1$.

Now one may ask the following question: Is it possible in any way to relax the nature of sharing the value 1 in Theorems $\mathrm{D}$ and $\mathrm{E}$ ?

The purpose of the paper is to study this problem. We shall not only relax the nature of sharing the value 1 but also weaken the condition on deficiencies. To this end we consider a gradation of sharing of values which measures how close a shared value is to being shared IM or being shared $\mathrm{CM}$ and is called weighted sharing of values as introduced in $[4,5]$.

Definition 1. Let $k$ be a nonnegative integer or infinity. For $a \in \mathbb{C} \cup$ $\{\infty\}$ we denote by $E_{k}(a ; f)$ the set of all $a$-points of $f$ where an $a$-point of multiplicity $m$ is counted $m$ times if $m \leq k$ and $k+1$ times if $m>k$.

If $E_{k}(a ; f)=E_{k}(a ; g)$, we say that $f, g$ share the value $a$ with weight $k$.

The definition implies that if $f, g$ share a value $a$ with weight $k$ then $z_{0}$ is a zero of $f-a$ with multiplicity $m(\leq k)$ if and only if $z_{0}$ is a zero of $g-a$ with multiplicity $m(\leq k)$, and $z_{0}$ is a zero of $f-a$ with multiplicity $m(>k)$ if and only if $z_{0}$ is a zero of $g-a$ with multiplicity $n(>k)$ where $m$ is not necessarily equal to $n$.

We write " $f, g$ share $(a, k)$ " to mean that $f, g$ share the value $a$ with weight $k$. Clearly if $f, g$ share $(a, k)$ then $f, g$ share $(a, p)$ for any integer $p$, $0 \leq p<k$. Also we note that $f, g$ share a value $a$ IM or CM if and only if $f, g$ share $(a, 0)$ or $(a, \infty)$ respectively.

Definition 2. We denote by $N(r, a ; f \mid=1)$ the counting function of simple $a$-points of $f$. 
Definition 3. If $s$ is a positive integer, we denote by $\bar{N}(r, a ; f \mid \geq s)$ the counting function of those $a$-points of $f$ whose multiplicities are greater than or equal to $s$, where each $a$-point is counted only once.

Definition 4. Let $f, g$ share a value $a \mathrm{IM}$. We denote by $\bar{N}_{*}(r, a ; f, g)$ the counting function of those $a$-points of $f$ whose multiplicities are not equal to multiplicities of the corresponding $a$-points of $g$, where each $a$-point is counted only once.

Clearly $\bar{N}_{*}(r, a ; f, g) \equiv \bar{N}_{*}(r, a ; g, f)$.

Definition 5 (cf. [1]). For a meromorphic function $f$ we put

$$
\begin{array}{rlrl}
T_{0}(r, f) & =\int_{1}^{r} \frac{T(t, f)}{t} d t, & N_{0}(r, a ; f) & =\int_{1}^{r} \frac{N(t, a ; f)}{t} d t, \\
N_{k}^{0}(r, a ; f) & =\int_{1}^{r} \frac{N_{k}(t, a ; f)}{t} d t, & m_{0}(r, f)=\int_{1}^{r} \frac{m(t, f)}{t} d t, \\
S_{0}(r, f) & =\int_{1}^{r} \frac{S(t, f)}{t} d t . &
\end{array}
$$

Definition 6. If $f$ is a meromorphic function, we put, for $a \in \mathbb{C} \cup\{\infty\}$,

$$
\begin{aligned}
& \delta_{0}(a ; f)=1-\limsup _{r \rightarrow \infty} \frac{N_{0}(r, a ; f)}{T_{0}(r, f)}, \\
& \Theta_{0}(a ; f)=1-\limsup _{r \rightarrow \infty} \frac{\bar{N}_{0}(r, a ; f)}{T_{0}(r, f)}, \\
& \delta_{k}^{0}(a ; f)=1-\limsup _{r \rightarrow \infty} \frac{N_{k}^{0}(r, a ; f)}{T_{0}(r, f)} .
\end{aligned}
$$

2. Lemmas. In this section we present some lemmas which will be needed in what follows. Let $f, g$ be two nonconstant meromorphic functions and we put

$$
h=\left(\frac{f^{\prime \prime}}{f^{\prime}}-\frac{2 f^{\prime}}{f-1}\right)-\left(\frac{g^{\prime \prime}}{g^{\prime}}-\frac{2 g^{\prime}}{g-1}\right) .
$$

LEMma 1. If $f, g$ share $(1,1)$ and $h \not \equiv 0$ then

(i) $N(r, 1 ; f \mid=1) \leq N(r, h)+S(r, f)+S(r, g)$,

(ii) $N(r, 1 ; g \mid=1) \leq N(r, h)+S(r, f)+S(r, g)$.

Proof. Since $f, g$ share $(1,1)$, it follows that a simple 1-point of $f$ is a simple 1-point of $g$ and conversely. Let $z_{0}$ be a simple 1-point of $f$ and $g$. Then by a simple calculation we see that in some neighbourhood of $z_{0}$,

$$
h=\left(z-z_{0}\right) \phi(z)
$$

where $\phi$ is analytic at $z_{0}$. 
Hence by the first fundamental theorem and the Milloux theorem $[2$, p. 47] we get

$$
N(r, 1 ; f \mid=1) \leq N(r, 0 ; h) \leq N(r, h)+S(r, f)+S(r, g),
$$

which is (i).

Now (ii) follows from (i) because $N(r, 1 ; f \mid=1) \equiv N(r, 1 ; g \mid=1)$. This proves the lemma.

Lemma 2. Let $f, g$ share $(1,0)$ and $h \not \equiv 0$. Then for any number $b$ $(\neq 0,1, \infty)$,

$$
\begin{aligned}
N(r, h) \leq & \bar{N}(r, \infty ; f \mid \geq 2)+\bar{N}(r, 0 ; f \mid \geq 2)+\bar{N}(r, b ; f \mid \geq 2) \\
& +\bar{N}(r, \infty ; g \mid \geq 2)+\bar{N}(r, 0 ; g \mid \geq 2)+\bar{N}_{*}(r, 1 ; f, g) \\
& +\bar{N}_{\oplus}\left(r, 0 ; f^{\prime}\right)+\bar{N}_{\otimes}\left(r, 0 ; g^{\prime}\right),
\end{aligned}
$$

where $\bar{N}_{\oplus}\left(r, 0 ; f^{\prime}\right)$ is the reduced counting function of those zeros of $f^{\prime}$ which are not zeros of $f(f-1)(f-b)$, and $\bar{N}_{\otimes}\left(r, 0 ; g^{\prime}\right)$ is the reduced counting function of those zeros of $g^{\prime}$ which are not zeros of $g(g-1)$.

Proof. We can easily verify that possible poles of $h$ occur at (i) multiple zeros of $f, g$; (ii) multiple poles of $f, g$; (iii) zeros of $f-1, g-1$; (iv) multiple zeros of $f-b$; (v) zeros of $f^{\prime}$ which are not zeros of $f(f-1)(f-b)$; (vi) zeros of $g^{\prime}$ which are not zeros of $g(g-1)$.

Let $z_{0}$ be a zero of $f-1$ with multiplicity $m(\geq 1)$ and of $g-1$ with multiplicity $n(\geq 1)$. Then in some neighbourhood of $z_{0}$ we get

$$
h=\frac{(n-m) \psi}{z-z_{0}}+\phi
$$

where $\phi, \psi$ are analytic at $z_{0}$ and $\psi\left(z_{0}\right) \neq 0$.

This shows that if $m=n$ then $z_{0}$ is not a pole of $h$ and if $m \neq n$ then $z_{0}$ is a simple pole of $h$. Since all the poles of $h$ are simple, the lemma is proved.

Lemma 3. If $f, g$ share $(1,2)$ then

$$
\begin{aligned}
N_{\otimes}\left(r, 0 ; g^{\prime}\right)+\bar{N}(r, 1 ; g \mid \geq 2)+\bar{N}_{*}(r, 1 ; f, g) \\
\leq \bar{N}(r, \infty ; g)+\bar{N}(r, 0 ; g)+S(r, g),
\end{aligned}
$$

where $N_{\otimes}\left(r, 0 ; g^{\prime}\right)$ is the counting function of those zeros of $g^{\prime}$ which are not zeros of $g(g-1)$.

Proof. Since $f, g$ share $(1,2)$, it follows that $\bar{N}_{*}(r, 1 ; f, g) \leq \bar{N}(r, 1$; $g \mid \geq 3)$. So remembering the definition of $N_{\otimes}\left(r, 0 ; g^{\prime}\right)$ we get 
(1)

$$
\begin{aligned}
N_{\otimes}\left(r, 0 ; g^{\prime}\right)+\bar{N}(r, 1 ; g \mid \geq 2)+\bar{N}_{*}(r, 1 ; f, g) & \\
& +N(r, 0 ; g)-\bar{N}(r, 0 ; g) \\
\leq & N_{\otimes}\left(r, 0 ; g^{\prime}\right)+\bar{N}(r, 1 ; g \mid \geq 2)+\bar{N}(r, 1 ; g \mid \geq 3) \\
& +N(r, 0 ; g)-\bar{N}(r, 0 ; g) \\
\leq & N\left(r, 0 ; g^{\prime}\right) .
\end{aligned}
$$

By the first fundamental theorem and the Milloux theorem [2, p. 55] we get

$$
\begin{aligned}
N\left(r, 0 ; g^{\prime}\right) & \leq N\left(r, 0 ; g^{\prime} / g\right)+N(r, 0 ; g)-\bar{N}(r, 0 ; g) \\
& \leq N\left(r, g^{\prime} / g\right)+N(r, 0 ; g)-\bar{N}(r, 0 ; g)+S(r, g) \\
& =\bar{N}(r, \infty ; g)+\bar{N}(r, 0 ; g)+N(r, 0 ; g)-\bar{N}(r, 0 ; g)+S(r, g) \\
& =\bar{N}(r, \infty ; g)+N(r, 0 ; g)+S(r, g) .
\end{aligned}
$$

Now the lemma follows from (1) and (2).

Lemma 4 (see [1]). $\lim _{r \rightarrow \infty} S_{0}(r, f) / T_{0}(r, f)=0$ through all values of $r$.

Lemma 5 (see [3]). For $a \in \mathbb{C} \cup\{\infty\}, \delta(a ; f) \leq \delta_{0}(a ; f), \Theta(a ; f) \leq$ $\Theta_{0}(a ; f)$ and $\delta_{k}(a ; f) \leq \delta_{k}^{0}(a ; f)$.

Lemma 6 (see [3]).

$$
\begin{aligned}
& \text { (i) } \liminf _{r \rightarrow \infty} \frac{T_{0}(r, \Psi(D) f)}{T_{0}(r, f)} \geq \sum_{a \neq \infty} \delta_{p}^{0}(a ; f), \\
& \text { (ii) } \delta_{0}(0 ; \Psi(D) f) \geq \frac{\sum_{a \neq \infty} \delta_{0}(a ; f)}{1+p\left(1-\Theta_{0}(\infty ; f)\right)} .
\end{aligned}
$$

Lemma 7 (see [3]). If $\sum_{a \neq \infty} \delta_{p}^{0}(a ; f)>0$ then

$$
\Theta_{0}(\infty ; \Psi(D) f) \geq 1-\frac{1-\Theta_{0}(\infty ; f)}{\sum_{a \neq \infty} \delta_{p}^{0}(a ; f)}
$$

Lemma 8 (see [8]). If $f$ is transcendental then $\lim _{r \rightarrow \infty} T_{0}(r, f) /(\log r)^{2}$ $=\infty$ through all values of $r$.

3. The main result. In this section we discuss the main result of the paper.

TheOREM 1. Let $f, g$ be two meromorphic functions such that

(i) $\Psi(D) f, \Psi(D) g$ are transcendental and share $(1,2)$ and 
(ii)

$$
\begin{aligned}
\frac{\sum_{a \neq \infty} \delta(a ; f)}{1+p(1-\Theta(\infty ; f))}+ & \frac{\sum_{a \neq \infty} \delta(a ; g)}{1+p(1-\Theta(\infty ; g))} \\
& +\min \left\{\delta_{2}(b ; \Psi(D) f), \delta_{2}(b ; \Psi(D) g)\right\} \\
>1 & +\frac{2(1-\Theta(\infty ; f))}{\sum_{a \neq \infty} \delta_{p}(a ; f)}+\frac{2(1-\Theta(\infty ; g))}{\sum_{a \neq \infty} \delta_{p}(a ; g)}
\end{aligned}
$$

for some $b \neq 0,1, \infty, 1 / 2,2,-\omega,-\omega^{2}$, with $\sum_{a \neq \infty} \delta_{p}(a ; f)>0, \sum_{a \neq \infty} \delta_{p}(a ; g)$ $>0$ and $\omega$ being the imaginary cube root of unity.

Then either $[\Psi(D) f][\Psi(D) g] \equiv 1$ or $f-g \equiv s$, where $s=s(z)$ is a solution of the differential equation $\Psi(D) w=0$.

The following example shows that Theorem 1 is sharp.

EXAMPLE 2. Let $f=\frac{1}{2} e^{z}\left(e^{z}-1\right), g=\frac{1}{2} e^{-z}\left(\frac{1}{2}-\frac{1}{5} e^{-z}\right)$ and $\Psi(D)=$ $D^{2}-3 D$. Then $\Psi(D) f=e^{z}\left(1-e^{z}\right), \Psi(D) g=e^{-z}\left(1-e^{-z}\right), \sum_{a \neq \infty} \delta(a ; f)=$ $\sum_{a \neq \infty} \delta(a ; g)=1 / 2, \Theta(\infty ; f)=\Theta(\infty ; g)=1, \delta_{2}(b ; \Psi(D) f)=\delta_{2}(b ; \Psi(D) g)$ $=0$ for $b \neq 0, \infty$ and $\Psi(D) f, \Psi(D) g$ share $(1,2)$. It is easily seen that neither $[\Psi(D) f][\Psi(D) g] \equiv 1$ nor $f-g \equiv c_{1}-c_{2} e^{3 z}$ for any constants $c_{1}$ and $c_{2}$.

Proof of Theorem 1. Let $F=\Psi(D) f$ and $G=\Psi(D) g$. Then in view of Lemmas 5-7 condition (ii) implies

$$
\begin{aligned}
\delta_{0}(0 ; F)+\delta_{0}(0 ; G)+2 \Theta_{0}(\infty ; F)+ & 2 \Theta_{0}(\infty ; G) \\
& +\min \left\{\delta_{2}^{0}(b ; F), \delta_{2}^{0}(b ; G)\right\}>5 .
\end{aligned}
$$

We put

$$
H=\left(\frac{F^{\prime \prime}}{F^{\prime}}-\frac{2 F^{\prime}}{F-1}\right)-\left(\frac{G^{\prime \prime}}{G^{\prime}}-\frac{2 G^{\prime}}{G-1}\right) .
$$

Suppose $H \not \equiv 0$. Then by Lemmas $1-3$ we get

$$
\begin{aligned}
N(r, 1 ; F \mid=1) \leq & \bar{N}(r, \infty ; F \mid \geq 2)+\bar{N}(r, 0 ; F \mid \geq 2)+\bar{N}(r, b ; F \mid \geq 2) \\
& +\bar{N}(r, \infty ; G \mid \geq 2)+\bar{N}(r, 0 ; G \mid \geq 2)+\bar{N}_{\oplus}\left(r, 0 ; F^{\prime}\right) \\
& +\bar{N}(r, \infty ; G)+\bar{N}(r, 0 ; G)-\bar{N}(r, 1 ; G \mid \geq 2) \\
& +S(r, F)+S(r, G) .
\end{aligned}
$$

By the second fundamental theorem we get

$$
\begin{aligned}
2 T(r, F) \leq & \bar{N}(r, \infty ; F)+\bar{N}(r, 1 ; F)+\bar{N}(r, b ; F) \\
& +\bar{N}(r, 0 ; F)-N_{\oplus}\left(r, 0 ; F^{\prime}\right)+S(r, F),
\end{aligned}
$$

where $N_{\oplus}\left(r, 0 ; F^{\prime}\right)$ is the counting function of those zeros of $F^{\prime}$ which are not zeros of $F(F-1)(F-b)$.

Since $F, G$ share $(1,2)$, we see that

$$
\begin{aligned}
\bar{N}(r, 1 ; F) & =\bar{N}(r, 1 ; F \mid=1)+\bar{N}(r, 1 ; F \mid \geq 2) \\
& =\bar{N}(r, 1 ; F \mid=1)+\bar{N}(r, 1 ; G \mid \geq 2) .
\end{aligned}
$$


Since $N_{2}(r, \infty ; F) \leq 2 \bar{N}(r, \infty ; F)$ and $N_{2}(r, \infty ; G) \leq 2 \bar{N}(r, \infty ; G)$, we get from (4)-(6) on integration

$$
\begin{aligned}
2 T_{0}(r, F) \leq & N_{2}^{0}(r, 0 ; F)+N_{2}^{0}(r, b ; F)+N_{2}^{0}(r, 0 ; G)+2 \bar{N}_{0}(r, \infty ; F) \\
& +2 \bar{N}_{0}(r, \infty ; G)+S_{0}(r, F)+S_{0}(r, G) .
\end{aligned}
$$

Similarly we obtain

$$
\begin{aligned}
2 T_{0}(r, G) \leq & N_{2}^{0}(r, 0 ; F)+N_{2}^{0}(r, b ; G)+N_{2}^{0}(r, 0 ; G)+2 \bar{N}_{0}(r, \infty ; F) \\
& +2 \bar{N}_{0}(r, \infty ; G)+S_{0}(r, F)+S_{0}(r, G) .
\end{aligned}
$$

From (7) and (8) we get

$$
\begin{aligned}
2 T_{0}(r) \leq & N_{2}^{0}(r, 0 ; F)+N_{2}^{0}(r, 0 ; G)+N_{2}^{0}(r, b)+2 \bar{N}_{0}(r, \infty ; F) \\
& +2 \bar{N}_{0}(r, \infty ; G)+S_{0}(r, F)+S_{0}(r, G),
\end{aligned}
$$

where $T_{0}(r)=\max \left\{T_{0}(r, F), T_{0}(r, G)\right\}$ and $N_{2}^{0}(r, b)=\max \left\{N_{2}^{0}(r, b ; F)\right.$, $\left.N_{2}^{0}(r, b ; G)\right\}$.

Since (9) contradicts (3), it follows that $H \equiv 0$. Then

$$
F=\frac{A G+B}{C G+D}
$$

where $A, B, C, D$ are complex numbers such that $A D-B C \neq 0$.

In view of (10) we get

$$
T_{0}(r, F)=T_{0}(r, G)+O(\log r) .
$$

Now we consider the following cases.

CASE 1: $A C \neq 0$. Then

$$
F-\frac{A}{C}=\frac{B-\frac{A D}{C}}{C G+D}
$$

Subcase 1.1: $A / C \neq b$. Then by the second fundamental theorem we get on integration

$$
\begin{aligned}
2 T_{0}(r & F) \\
& \leq \bar{N}_{0}(r, \infty ; F)+\bar{N}_{0}(r, 0 ; F)+\bar{N}_{0}(r, A / C ; F)+\bar{N}_{0}(r, b ; F)+S_{0}(r, F) \\
& =\bar{N}_{0}(r, \infty ; F)+\bar{N}_{0}(r, 0 ; F)+\bar{N}_{0}(r, b ; F)+\bar{N}_{0}(r, \infty ; G)+S_{0}(r, F),
\end{aligned}
$$

which implies (9) in view of (11) and Lemma 8 and finally contradicts (3).

Subcase 1.2: $A / C=b$. Also we suppose that $B D \neq 0$. Then $B / D \neq b$ because $A D-B C \neq 0$. So by the second fundamental theorem we get on integration

$$
\begin{aligned}
2 T_{0}(r, & F) \\
& \leq \bar{N}_{0}(r, \infty ; F)+\bar{N}_{0}(r, 0 ; F)+\bar{N}_{0}(r, b ; F)+\bar{N}_{0}(r, B / D ; F)+S_{0}(r, F) \\
& =\bar{N}_{0}(r, \infty ; F)+\bar{N}_{0}(r, 0 ; F)+\bar{N}_{0}(r, b ; F)+\bar{N}_{0}(r, 0 ; G)+S_{0}(r, F),
\end{aligned}
$$

which by (11) and Lemma 8 implies (9) and so contradicts (3). 
Let $B=0$. Then $D \neq 0$ because $F$ is nonconstant. Now from (12) we get

$$
F-b=\frac{-b}{\alpha G+1}
$$

where $\alpha=C / D$.

Let 1 be a Picard exceptional value (e.v.P.) of $F$ and so of $G$. Then by the second fundamental theorem we get on integration

$$
2 T_{0}(r, F) \leq \bar{N}_{0}(r, \infty ; F)+\bar{N}_{0}(r, 0 ; F)+\bar{N}_{0}(r, b ; F)+S_{0}(r, F),
$$

which implies (9) in view of (11) and Lemma 8 and so contradicts (3). that

Let 1 be not an e.v.P. of $F$ and $G$. Then from (13) we get $\alpha=\frac{1}{b-1}$ so

$$
F=\frac{b G}{(b-1)+G} .
$$

Since $b \neq 1 / 2$, by the second fundamental theorem we get on integration $2 T_{0}(r, G)$

$$
\begin{aligned}
& \leq \bar{N}_{0}(r, \infty ; G)+\bar{N}_{0}(r, 0 ; G)+\bar{N}_{0}(r, b ; G)+\bar{N}_{0}(r, 1-b ; G)+S_{0}(r, G) \\
& =\bar{N}_{0}(r, \infty ; G)+\bar{N}_{0}(r, 0 ; G)+\bar{N}_{0}(r, b ; G)+\bar{N}_{0}(r, \infty ; F)+S_{0}(r, G),
\end{aligned}
$$

which by (11) and Lemma 8 implies (9) and so contradicts (3).

Let $B \neq 0, D=0$. Then from (12) we obtain

$$
F=b+\frac{\beta}{G},
$$

where $\beta=B / C$.

If 1 is an e.v.P. of $F$ and so of $G$, by the second fundamental theorem we get on integration

$$
2 T_{0}(r, F) \leq \bar{N}_{0}(r, \infty ; F)+\bar{N}_{0}(r, 0 ; F)+\bar{N}_{0}(r, b ; F)+S_{0}(r, F),
$$

which implies (9) in view of (11) and Lemma 8 and so contradicts (3).

Suppose 1 is not an e.v.P. of $F$ and $G$. Then from (14) we get $\beta=1-b$ so that

$$
F=b+\frac{1-b}{G} .
$$

Since $b \neq-\omega,-\omega^{2}$, by the second fundamental theorem we get on integration

$$
\begin{aligned}
& 2 T_{0}(r, G) \\
& \quad \leq \bar{N}_{0}(r, \infty ; G)+\bar{N}_{0}(r, 0 ; G)+\bar{N}_{0}(r, b ; G)+\bar{N}_{0}(r, 1-1 / b ; G)+S_{0}(r, G) \\
& \quad=\bar{N}_{0}(r, \infty ; G)+\bar{N}_{0}(r, 0 ; G)+\bar{N}_{0}(r, b ; G)+\bar{N}_{0}(r, 0 ; F)+S_{0}(r, G),
\end{aligned}
$$

which implies (9) in view of (11) and Lemma 8 and so contradicts (3). 
CASE 2: $A C=0$. Since $F$ is nonconstant, it follows that $A$ and $C$ are not simultaneously zero.

SubCASE 2.1: $A=0$ and $C \neq 0$. Then $B \neq 0$ and from (10) we get

$$
\frac{1}{F}=\alpha G+\beta,
$$

where $\alpha=C / B$ and $\beta=D / B$.

If 1 is an e.v.P. of $F$ and $G$, by the second fundamental theorem we get on integration

$$
2 T_{0}(r, F) \leq \bar{N}_{0}(r, \infty ; F)+\bar{N}_{0}(r, 0 ; F)+\bar{N}_{0}(r, b ; F)+S_{0}(r, F),
$$

which by (11) and Lemma 8 implies (9) and so contradicts (3).

Suppose 1 is not an e.v.P. of $F$ and $G$. Then from (15) we get $\alpha+\beta=1$ so that

$$
\frac{1}{F}=\alpha G+1-\alpha .
$$

If $\alpha \neq 1,1-1 / b$, by the second fundamental theorem we get on integration $2 T_{0}(r, F)$

$$
\begin{aligned}
& \leq \bar{N}_{0}(r, \infty ; F)+\bar{N}_{0}(r, 0 ; F)+\bar{N}_{0}(r, b ; F)+\bar{N}_{0}(r, 1 /(1-\alpha) ; F)+S_{0}(r, F) \\
& =\bar{N}_{0}(r, \infty ; F)+\bar{N}_{0}(r, 0 ; F)+\bar{N}_{0}(r, b ; F)+\bar{N}_{0}(r, 0 ; G)+S_{0}(r, F),
\end{aligned}
$$

which implies (9) in view of (11) and Lemma 8 and so contradicts (3).

If $\alpha=1$ then $F G \equiv 1$, i.e. $[\Psi(D) f][\Psi(D) g] \equiv 1$.

If $\alpha=1-1 / b$ then

$$
F=\frac{b}{1+(b-1) G} .
$$

Since $b \neq-\omega,-\omega^{2}$, by the second fundamental theorem we get on integration

$$
\begin{aligned}
& 2 T_{0}(r, G) \\
& \leq \bar{N}_{0}(r, \infty ; G)+\bar{N}_{0}(r, 0 ; G)+\bar{N}_{0}(r, b ; G)+\bar{N}_{0}(r, 1 /(1-b) ; G)+S_{0}(r, G) \\
& =\bar{N}_{0}(r, \infty ; G)+\bar{N}_{0}(r, 0 ; G)+\bar{N}_{0}(r, b ; G)+\bar{N}_{0}(r, \infty ; F)+S_{0}(r, G),
\end{aligned}
$$

which by (11) and Lemma 8 implies (9) and so contradicts (3).

Subcase 2.2: $A \neq 0$ and $C=0$. Then $D \neq 0$ and from (10) we get

$$
F=\alpha G+\beta,
$$

where $\alpha=A / D, \beta=B / D$.

If 1 is an e.v.P. of $F$ and $G$, by the second fundamental theorem we get on integration

$$
2 T_{0}(r, F) \leq \bar{N}_{0}(r, \infty ; F)+\bar{N}_{0}(r, 0 ; F)+\bar{N}_{0}(r, b ; F)+S_{0}(r, F),
$$

which implies (9) by (11) and Lemma 8 and so contradicts (3). 
Suppose 1 is not an e.v.P. of $F$ and $G$. Then from (16) we get $\alpha+\beta=1$ and so

$$
F=\alpha G+1-\alpha .
$$

If $\alpha \neq 1,1-b$, by the second fundamental theorem we get on integration $2 T_{0}(r, F)$

$$
\begin{aligned}
& \leq \bar{N}_{0}(r, \infty ; F)+\bar{N}_{0}(r, 0 ; F)+\bar{N}_{0}(r, b ; F)+\bar{N}_{0}(r, 1-\alpha ; F)+S_{0}(r, F) \\
& =\bar{N}_{0}(r, \infty ; F)+\bar{N}_{0}(r, 0 ; F)+\bar{N}_{0}(r, b ; F)+\bar{N}_{0}(r, 0 ; G)+S_{0}(r, F)
\end{aligned}
$$

which implies (9) in view of (11) and Lemma 8 and so contradicts (3).

If $\alpha=1$ then $F \equiv G$ and so $f-g \equiv s$, where $s=s(z)$ is a solution of the differential equation $\Psi(D) w=0$.

If $\alpha=1-b$ then

$$
F=(1-b) G+b .
$$

Since $b \neq 2$, by the second fundamental theorem we get on integration

$$
\begin{aligned}
& 2 T_{0}(r, G) \\
& \quad \leq \bar{N}_{0}(r, \infty ; G)+\bar{N}_{0}(r, 0 ; G)+\bar{N}_{0}(r, b ; G)+\bar{N}_{0}(r, b /(b-1) ; G)+S_{0}(r, G) \\
& =\bar{N}_{0}(r, \infty ; G)+\bar{N}_{0}(r, 0 ; G)+\bar{N}_{0}(r, b ; G)+\bar{N}_{0}(r, 0 ; F)+S_{0}(r, G),
\end{aligned}
$$

which by (11) and Lemma 8 implies (9) and so contradicts (3). This proves the theorem.

4. Applications. In this section we discuss two applications of the main theorem, the first of which improves a result of Yi and Yang [13] and the second gives a better answer to the question of Yang [9] mentioned in the introduction.

THEOREM 2. Let $f, g$ be two nonconstant meromorphic functions with $\Theta(\infty ; f)=\Theta(\infty ; g)=1$. If for $n \geq 1$ the derivatives $f^{(n)}, g^{(n)}$ share $(1,2)$ and

(i) $\sum_{a \neq \infty} \delta(a ; f)+\sum_{a \neq \infty} \delta(a ; g)+\min \left\{\delta_{2}\left(b ; f^{(n)}\right), \delta_{2}\left(b ; g^{(n)}\right)\right\}>1$

for some $b \neq 0,1, \infty, 1 / 2,2,-\omega,-\omega^{2}$, and

(ii) $\Theta(\alpha ; f)+\Theta(\alpha ; g)>1$

for some $\alpha \neq \infty$, then either (I) $f^{(n)} g^{(n)} \equiv 1$ or (II) $f \equiv g$.

Proof. From the given condition it follows that $f, g$ are transcendental and so $f^{(n)}, g^{(n)}$ are transcendental. Choosing $\Psi(D)=D^{n}$ in Theorem 1 we get either $f^{(n)} g^{(n)} \equiv 1$ or $f-g \equiv Q$, where $Q$ is a polynomial of degree at most $n-1$. If possible let $Q \not \equiv 0$. Then by Nevanlinna's theorem on three 
small functions $[2$, p. 47$]$ we get

$$
\begin{aligned}
T(r, f) & \leq \bar{N}(r, \alpha ; f)+\bar{N}(r, \alpha+Q ; f)+\bar{N}(r, \infty ; f)+S(r, f) \\
& =\bar{N}(r, \alpha ; f)+\bar{N}(r, \alpha ; g)+\bar{N}(r, \infty ; f)+S(r, f) .
\end{aligned}
$$

Since $f-g \equiv Q$, it follows that $T(r, f)=T(r, g)+O(\log r)$. So $\Theta(\alpha ; f)+$ $\Theta(\alpha ; g) \leq 1$, which is a contradiction. Therefore $Q \equiv 0$ and so $f \equiv g$. This proves the theorem.

The following examples show that the condition $\Theta(\alpha ; f)+\Theta(\alpha ; g)>1$ is necessary for the validity of case (II).

EXAMPle 3. Let $f=1+e^{z}$ and $g=e^{z}$. Then

$$
\sum_{a \neq \infty} \delta(a ; f)+\sum_{a \neq \infty} \delta(a ; g)+\min \left\{\delta_{2}\left(b ; f^{(n)}\right), \delta_{2}\left(b ; g^{(n)}\right)\right\}=2
$$

for any $b \neq 0, \infty, \Theta(\infty ; f)=\Theta(\infty ; g)=1, \Theta(0 ; f)+\Theta(0 ; g)=1, \Theta(1 ; f)+$ $\Theta(1 ; g)=1, \Theta(\alpha ; f)+\Theta(\alpha ; g)<1$ for $\alpha \neq 0,1, \infty$ and $f^{(n)}, g^{(n)}$ share $(1,2)$ but $f-g \equiv 1$.

ExAmple 4. Let $f=1+e^{z}$ and $g=(-1)^{n} e^{-z}$. Then $\sum_{a \neq \infty} \delta(a ; f)+$ $\sum_{a \neq \infty} \delta(a ; g)+\min \left\{\delta_{2}\left(b ; f^{(n)}\right), \delta_{2}\left(b ; g^{(n)}\right)\right\}=2$ for any $b \neq 0, \infty, \Theta(\infty ; f)=$ $\Theta(\infty ; g)=1, \Theta(0 ; f)+\Theta(0 ; g)=1, \Theta(1 ; f)+\Theta(1 ; g)=1, \Theta(\alpha ; f)+\Theta(\alpha ; g)$ $<1$ for $\alpha \neq 0,1, \infty$ and $f^{(n)}, g^{(n)}$ share $(1,2)$ but $f^{(n)} g^{(n)} \equiv 1$.

REMARK 1. Theorem 2 improves Theorem C, a result of Yi and Yang [13] and also a recent result of Lahiri [3].

In the following theorem we provide a better answer to a question of Yang [9] than those given in Theorems F and G.

THEOREM 3. Let $f$ and $g$ be two meromorphic functions such that $f^{(n)}$, $g^{(n)}(n \geq 1)$ share $(1,2), f, g$ share $(\alpha, 0)$ for some $\alpha \neq \infty$ and

$$
\begin{array}{r}
\frac{\sum_{a \neq \infty} \delta(a ; f)}{1+p(1-\Theta(\infty ; f))}+\frac{\sum_{a \neq \infty} \delta(a ; g)}{1+p(1-\Theta(\infty ; g))}+\min \left\{\delta_{2}\left(b ; f^{(n)}\right), \delta_{2}\left(b ; g^{(n)}\right)\right\} \\
>1+\frac{2(1-\Theta(\infty ; f))}{\sum_{a \neq \infty} \delta_{p}(a ; f)}+\frac{2(1-\Theta(\infty ; g))}{\sum_{a \neq \infty} \delta_{p}(a ; g)}
\end{array}
$$

for some $b \neq 0,1, \infty, 1 / 2,2,-\omega,-\omega^{2}$, with $\sum_{a \neq \infty} \delta_{p}(a ; f)>0, \sum_{a \neq \infty} \delta_{p}(a ; g)$ $>0$ and $\omega$ being the imaginary cube root of unity. Then either $f^{(n)} g^{(n)} \equiv 1$ or $f \equiv g$.

Proof. From the assumption it follows that $f$ and $g$ are transcendental and so $f^{(n)}$ and $g^{(n)}$ are transcendental. Choosing $\Psi(D)=D^{n}$ we see from Theorem 1 that either $f-g \equiv Q$ or $f^{(n)} g^{(n)} \equiv 1$, where $Q$ is a polynomial of degree at most $n-1$. If possible, let $Q \not \equiv 0$. Since $f, g$ share $(\alpha, 0)$, it follows 
that $\bar{N}(r, \alpha ; f)=\bar{N}(r, \alpha ; g) \leq \bar{N}(r, 0 ; Q)=O(\log r)$. Now by Nevanlinna's theorem on three small functions $[2$, p. 47] we get

$$
\begin{aligned}
T(r, f) & \leq \bar{N}(r, \alpha ; f)+\bar{N}(r, \alpha+Q ; f)+\bar{N}(r, \infty ; f)+S(r, f) \\
& =\bar{N}(r, \alpha ; f)+\bar{N}(r, \alpha ; g)+\bar{N}(r, \infty ; f)+S(r, f) \\
& =\bar{N}(r, \infty ; f)+O(\log r)+S(r, f),
\end{aligned}
$$

which implies that $\Theta(\infty ; f)=0$. Similarly we see that $\Theta(\infty ; g)=0$. Since this contradicts the assumption, it follows that $Q \equiv 0$ and so $f \equiv g$. This proves the theorem.

The following example shows that Theorem 3 is sharp.

EXAMPLE 5. Let $f=-2^{-n} e^{2 z}+(-1)^{n+1} 2^{-n} e^{z}$ and $g=(-1)^{n+1} 2^{-n} e^{-2 z}$ $-2^{-n} e^{-z}$. Then $f^{(n)}, g^{(n)}$ share $(1,2), f, g$ share $(0,0), \Theta(\infty ; f)=\Theta(\infty ; g)$ $=1$ and $\sum_{a \neq \infty} \delta(a ; f)+\sum_{a \neq \infty} \delta(a ; g)+\min \left\{\delta_{2}\left(b ; f^{(n)}\right), \delta_{2}\left(b ; b^{(n)}\right)\right\}=1$ for any $b \neq 0, \infty$ but neither $f \equiv g$ nor $f^{(n)} g^{(n)} \equiv 1$.

Concluding Remark. Since Example 1 shows that in Theorem 1 sharing $(1,2)$ cannot be relaxed to sharing $(1,0)$, we conclude the paper with the following question: Is it possible in Theorem 1 to relax sharing $(1,2)$ to sharing $(1,1)$ ?

Acknowledgements. The author is grateful to the referee for valuable comments.

\section{References}

[1] M. Furuta and N. Toda, On exceptional values of meromorphic functions of divergence class, J. Math. Soc. Japan 25 (1973), 667-679.

[2] W. K. Hayman, Meromorphic Functions, Clarendon Press, Oxford, 1964.

[3] I. Lahiri, Uniqueness of meromorphic functions when two linear differential polynomials share the same 1-points, Ann. Polon. Math. 71 (1999), 113-128.

[4] -, Weighted sharing and uniqueness of meromorphic functions, Nagoya Math. J. 161 (2001), 193-206.

[5] - Weighted value sharing and uniqueness of meromorphic functions, Complex Variables 46 (2001), 241-253.

[6] E. Mues and M. Reinders, On a question of C. C. Yang, ibid. 34 (1997), 171-179.

[7] K. Shibazaki, Unicity theorems for entire functions of finite order, Mem. National Defence Acad. Japan 21 (1981), no. 3, 67-71.

[8] N. Toda, On a modified deficiency of meromorphic functions, Tôhoku Math. J. 22 (1970), 635-658.

[9] C. C. Yang, On two entire functions which together with their first derivatives have the same zeros, J. Math. Anal. Appl. 56 (1976), 1-6.

[10] H. X. Yi, A question of C. C. Yang on the uniqueness of entire functions, Kodai Math. J. 13 (1990), 39-46.

[11] -, Uniqueness of meromorphic functions and a question of C. C. Yang, Complex Variables 14 (1990), 169-174. 
[12] H. X. Yi, Unicity theorems for entire or meromorphic functions, Acta Math. Sinica (N.S.) 10 (1994), 121-131.

[13] H. X. Yi and C. C. Yang, A uniqueness theorem for meromorphic functions whose nth derivatives share the same 1-points, J. Anal. Math. 62 (1994), 261-270.

Department of Mathematics

University of Kalyani

West Bengal 741235, India

E-mail: indrajit@cal2.vsnl.net.in

Reçu par la Rédaction le 5.9.2001

Révisé le 9.3.2002 